\section{Quemadura eléctrica en el pene tratada con penectomía parcial. Reporte de un caso}

Piñón-Solís EO, Morales-Ordaz O, Gil-García JF, Lujano-Pedraza $\mathrm{H}$, ValdezColín JA, Noyola-Guadarrama A, Michel-Ramírez JM

\section{Resumen}

ANTECEDENTES: las quemaduras eléctricas de alto voltaje en el pene son sucesos poco frecuentes, asociados con alta morbilidad y mortalidad, que pueden causar lesiones devastadoras y afectar la función eréctil, calidad de vida y aspecto psicológico del paciente.

CASO CLÍNICO: paciente de 28 años de edad, atendido en el área de choque del Centro Médico Adolfo López Mateos (ISEM) por quemadura eléctrica con expansión hacia los genitales. A la exploración física se observaron lesiones en el antebrazo derecho y el pene, con necrosis y pérdida de la función de la uretra, cuerpos cavernosos y esponjosos en 2 tercios distales y la formación de un "globo" vesical con retención aguda de orina. Se decidió la cistostomía suprapúbica, debridación extensa del tejido necrótico y penectomía parcial, sin incidencias posoperatorias. El paciente se encuentra en protocolo de reconstrucción peneana anatómica y preservación de la función miccional.

CONCLUSIONES: las quemaduras eléctricas en los genitales son sucesos excepcionales. Entre las opciones de tratamiento quirúrgicourológico más importantes se encuentran la preservación y recuperación de funcionalidad del pene en los pacientes jóvenes.

PALABRAS CLAVE: quemadura eléctrica, penectomía parcial, tratamiento por quemadura eléctrica.

Rev Mex Urol. 2017 Sep-Oct;77(5):401-404.

\section{Electrical burn affecting the penis managed through partial penectomy: A case report}

Piñón-Solís EO, Morales-Ordaz O, Gil-García JF, Lujano-Pedraza $\mathrm{H}$, ValdezColín JA, Noyola-Guadarrama A, Michel-Ramírez JM

\section{Abstract}

BACKGROUND: High-voltage electrical burns are uncommon and can cause multisystemic lesions with high morbidity and mortality. When they involve the penis, they are devastating, affecting erectile function, quality of life, and the psychologic aspect of the patient.
Departamento de Urología, Centro Médico Adolfo López Mateos, Instituto de Salud del Estado de México, Toluca.

Recibido: diciembre 2016

Aceptado: diciembre 2016

Correspondencia

Enrique Omar Piñón Solis

eopinon@hotmail.com

Este artículo debe citarse como

Piñón-Solís EO, Morales-Ordaz O, Gil-García JF, Lujano-Pedraza H, Valdez-Colín JA, Noyola-Guadarrama A, Michel-Ramírez JM. Quemadura eléctrica en el pene tratada con penectomía parcial. Reporte de un caso. Rev Mex Urol. 2017 sep-oct;77(5):401-404. DOI: https://doi.org/10.24245/revmexurol.v77i5.1116 
CLINICAL CASE: A 28-year-old man was brought to the emergency room of the Centro Médico Adolfo López Mateos (ISEM) with a highvoltage electrical burn affecting the genitals. Physical examination revealed lesions in the right forearm and the penis, with necrosis and loss of function of the urethra, corpora cavernosa, and corpus spongiosum at the distal two thirds, and bladder distension with acute urinary retention. Suprapubic cystostomy, extensive debridement of the necrotic tissue, and partial penectomy were performed, with no postoperative incidents. The patient is currently under the treatment protocol for anatomic penile reconstruction and voiding function preservation.

CONCLUSIONS: Electrical burns affecting the genitals are exceptional. Penile preservation and functional recovery are among the most important surgical-urologic treatment options in young patients.

KEYWORDS: Electrical burn; Partial penectomy; Electrical burn management
Departamento de Urología, Centro Médico Adolfo López Mateos, Instituto de Salud del Estado de México, Toluca.

Correspondence

Enrique Omar Piñón Solis eopinon@hotmail.com

\section{ANTECEDENTES}

Las quemaduras eléctricas de alto voltaje son poco frecuentes, pero pueden causar lesiones multisistémicas con alta tasa de morbilidad y mortalidad. Las quemaduras eléctricas en el pene son excepcionales y devastadoras, pues afectan la función eréctil, calidad de vida y aspecto psicológico del paciente.

\section{CASO CLÍNICO}

Paciente de 28 años de edad, sin antecedentes heredofamiliares de importancia para el padecimiento actual, atendido en el área de choque del Centro Médico Adolfo López Mateos (ISEM) por quemadura eléctrica con expansión hacia los genitales, al tener contacto con cables de alta tensión mientras pintaba un tubo en un andamio. Recibió la descarga eléctrica en el tórax, en el lado derecho, con pérdida inmediata del estado de alerta. A su ingreso al servicio de Urgencias refirió dolor en las extremidades y en el antebrazo derecho; a la exploración física se observaron lesiones por quemadura eléctrica en el antebrazo derecho y el pene, con necrosis y pérdida de la función de la uretra, cuerpos cavernosos y esponjosos en 2 tercios distales del pene (Figura 1), y la formación de un "globo" vesical con retención aguda de orina. Los estudios de laboratorio reportaron leucocitos 9500, hemoglobina $13.9 \mathrm{mg} / \mathrm{dL}$, glucosa $116 \mathrm{~g} / \mathrm{dL}$, urea $94 \mathrm{mg} / \mathrm{dL}$, creatinina $0.8 \mathrm{mg} / \mathrm{dL}$; examen general de orina con hemoglobinuria y mioglobinuria, y concentraciones elevadas de enzimas musculares.

Se decidió efectuar cistostomía suprapúbica, debridación extensa del tejido necrótico y penectomía parcial (Figura 2), sin incidencias a corto plazo. Hoy día el paciente se encuentra en protocolo de reconstrucción anatómica del pene y preservación de la función miccional.

\section{DISCUSIÓN}

Las quemaduras en los genitales son excepcionales, pues su incidencia estimada es de 2.7 a 


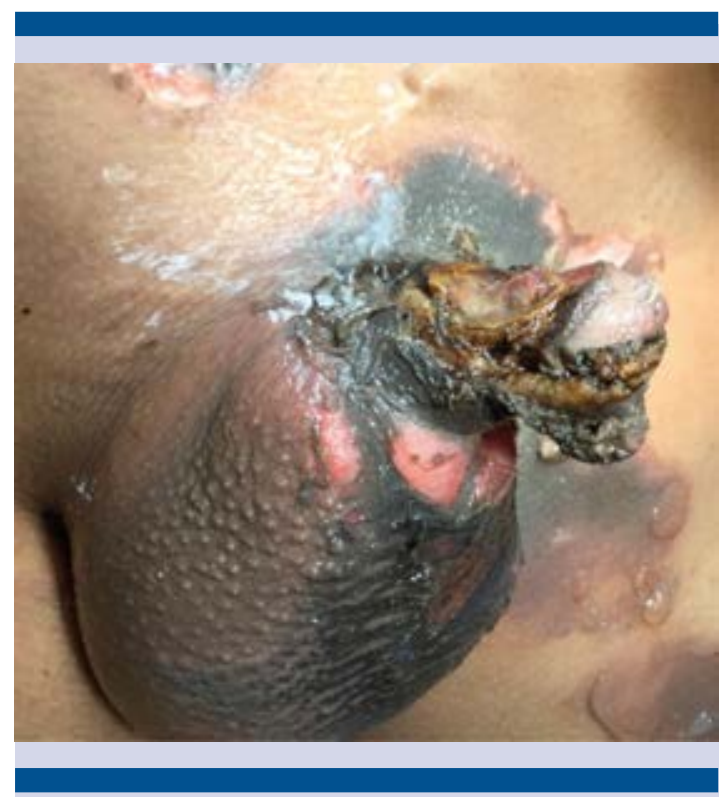

Figura 1. Quemadura eléctrica en el pene.

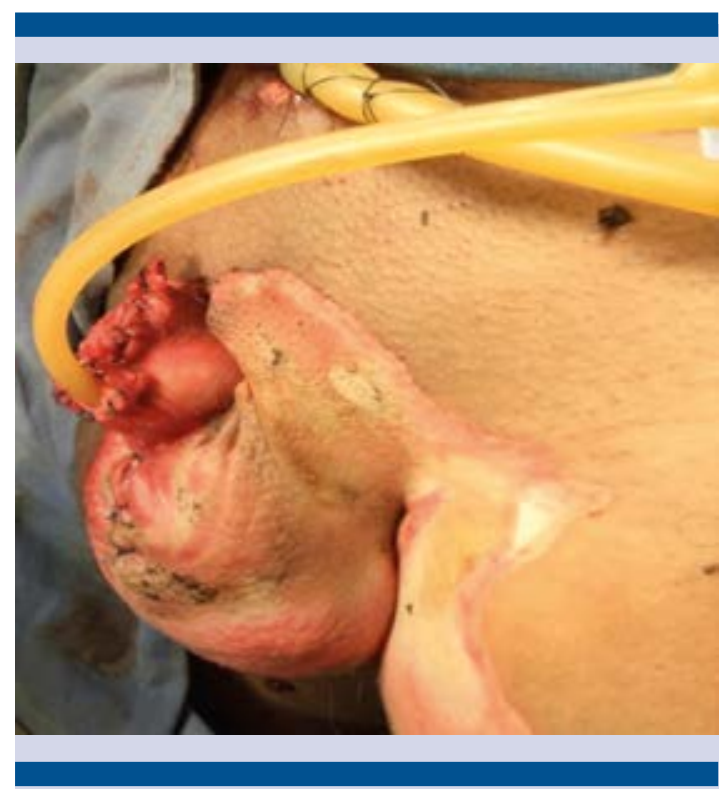

Figura 2. Penectomía parcial.

$12.5 \%$, según el tipo y localización, ${ }^{1}$ y la mortalidad de 2.7 a $67 \%$. Este tipo de sucesos provocan la muerte en más de 500 personas al año en Estados Unidos. ${ }^{2}$ Las quemaduras eléctricas ocupan el segundo lugar en prevalencia, entre las térmicas y las químicas. ${ }^{1}$ El tratamiento conservador en este tipo de pacientes es raro. ${ }^{1}$ Estas lesiones son frecuentes en trabajadores eléctricos y su severidad depende de la duración y región corporal afectada, incluso puede provocar la muerte inmediata por fibrilación ventricular o asistolia y paro respiratorio por parálisis del control central respiratorio, con lesiones y necrosis miocárdica y al sistema nervioso central. ${ }^{2}$

Cualquier material que altere el voltaje genera resistencia por conducción o por contacto directo; de esta manera, las quemaduras eléctricas pueden originarse por ambos mecanismos (directos e indirectos), por ejemplo: el daño es causado por alguna lesión directa o por la conversión de energía eléctrica en energía térmica, que es responsable de la lesión secundaria. Las lesiones son proporcionales al voltaje e inversamente a la resistencia del conductor. La resistencia de los nervios, vasos sanguíneos, mucosas y músculos son relativamente bajas y, por lo tanto, son excelentes conductores de energía (debido a alto contenido en agua); el hueso, la grasa y los tendones muestran resistencia relativamente alta y la piel implica resistencia intermedia. ${ }^{2}$

Robson y sus colaboradores describieron la fisiopatología de la influencia vascular de las quemaduras por electricidad al paso de la corriente eléctrica, produciendo metabolitos de ácido araquidónico para generar contracción vascular y trombosis; sus resultados mostraron que las lesiones dependen del tamaño de los vasos, por ejemplo, los vasos pequeños son más susceptibles al alto voltaje que los vasos grandes. ${ }^{2}$

La amputación del pene por quemaduras eléctricas resulta psicológicamente devastadora, por lo que la mayoría de los pacientes requiere tratamiento multidisciplinario. ${ }^{3}$ Este tipo de accidentes son poco frecuentes, así lo demuestra el estudio Shameemshah, en la India, ${ }^{4}$ quien 
reportó 5 casos en 10 años. Los pacientes con este tipo de quemaduras tienen alteraciones psicoafectivas, caracterizadas por depresión, ansiedad y otros trastornos orgánicos, propios de la lesión, como disfunción eréctil. Salehi y su grupo, quienes utilizaron el International Index of Erectile Function (IIEF-5), y Kim y sus coautores, reportaron $53 \%$ de pacientes con disfunción eréctil ${ }^{5}$ y alta tasa de infección por riesgo elevado de contaminación urinaria y fecal. ${ }^{2}$

El diagnóstico y tratamiento de pacientes con lesiones genitales por quemaduras eléctricas se establece mediante examen físico, estudios de laboratorio, profilaxis tetánica, prescripción de antibióticos intravenosos y estimación de la lesión por la quemadura. En estos pacientes debe examinarse la vejiga y el recto mediante cistoscopia y sigmoidoscopia (si fuera aplicable), además del posible daño esquelético. El uroanálisis está indicado para determinar algún daño renal con mioglobinuria o hemoglobinuria. Además, el paciente debe hidratarse intensamente para alcalinizar la orina. También están indicados el monitoreo cardiaco y el tratamiento quirúrgico. ${ }^{6}$

En general, las recomendaciones para el tratamiento quirúrgico en pacientes con quemaduras térmicas, eléctricas o químicas son la exploración y desbridamiento, este último limitado al tejido no viable en pacientes con pérdida pérdida extensa de la piel genital o lesiones complicadas con infección. ${ }^{7}$ Estas lesiones suelen ser extensas, por lo que debe colocarse un catéter suprapúbico, sobre todo en los casos que llega a conservarse el pene; sin embargo, según la historia natural de las lesiones por quemaduras eléctricas, pueden terminar en autoamputación, con extensión a los tejidos profundos y destrucción. ${ }^{8}$ Debido a esto, el tratamiento inicial incluye: exploración quirúrgica, irrigación y desbridamiento limitado, además de la eliminación de tejido no viable visible. Estas lesiones suelen requerir múltiples intervenciones reconstructivas, prescripción de antibióticos tópicos y uso de dispositivos de vacío con presión negativa.?

Las quemaduras eléctricas en los genitales son sucesos excepcionales que afectan la calidad de vida de los pacientes. Entre las opciones de tratamiento quirúrgico-urológico más importantes se encuentran la preservación y recuperación de la funcionalidad del pene en los pacientes jóvenes.

\section{Conflictos de interés}

Los autores declaran no tener conflictos de interés.

\section{Fuente de financiamiento}

Los autores no recibieron ningún patrocinio para llevar a cabo este estudio.

\section{REFERENCIAS}

1. Harpole BG, Wibbenmeyer LA, Erickson BA. Genital burning the national burn repository: incidence, etiology and impact on morbidity and mortality. Urology 2014;83:298-303.

2. Kim HJ, Chol SH, Shin TS, et al. Erectile dysfunction in patients with electrical injury. Urology 2007;70:1200-1203.

3. Housinger TA, Keller B, Warden GD. Management of burns of the penis. J Burn Care Rehabil 1993;14(5):535-7.

4. Prakash V. Amputation of the penis due to electrical burnrole of prefabricated urethra in penile reconstruction. Burns 2008;34:119-21.

5. Salehi SH, As'di K, Naderan M, et al. Assessment of erectile dysfunction following burn injury. Urology 2016; 93:112-116

6. Chang AJ, Brandes SB. Advances in diagnosis and management of genital injuries. Urol Clin N Am 40 2013:427-438.

7. Morey AF et al. Urotrauma: AUA Guideline. J Urol 2014;192:327-335.

8. Medendorp AR, Albrecht MC, Morey AF. Natural history of extensive, full-thickness electrical burns involving the penis. Urology 2007;70(3):588-589. 